\title{
Multiplex evolution of antibody fragments utilizing a yeast surface display platform
}

\author{
Oh, Eun Joong; Liu, Rongming; Liang, Liya; Freed, Emily F.; Eckert, Carrie Ann; Gill, Ryan T.
}

\section{Published in:}

ACS Synthetic Biology

Link to article, DOI:

10.1021/acssynbio.0c00159

Publication date:

2020

Document Version

Peer reviewed version

Link back to DTU Orbit

Citation (APA):

Oh, E. J., Liu, R., Liang, L., Freed, E. F., Eckert, C. A., \& Gill, R. T. (2020). Multiplex evolution of antibody fragments utilizing a yeast surface display platform. ACS Synthetic Biology, 9(8), 2197-2202. https://doi.org/10.1021/acssynbio.0c00159

\section{General rights}

Copyright and moral rights for the publications made accessible in the public portal are retained by the authors and/or other copyright owners and it is a condition of accessing publications that users recognise and abide by the legal requirements associated with these rights.

- Users may download and print one copy of any publication from the public portal for the purpose of private study or research.

- You may not further distribute the material or use it for any profit-making activity or commercial gain

- You may freely distribute the URL identifying the publication in the public portal 


\title{
Multiplex Evolution of Antibody Fragments Utilizing a Yeast Surface Display Platform
}

\author{
Eun Joong Oh, Rongming Liu, Liya Liang, Emily F. Freed, Carrie A. Eckert, and Ryan T. Gill*
}

Cite This: https://dx.doi.org/10.1021/acssynbio.0c00159

Read Online

ABSTRACT: Advances in high-throughput synthetic biology technologies based on the CRISPR/Cas9 system have enabled a comprehensive assessment of mutations conferring desired phenotypes, as well as a better understanding of genotypephenotype correlations in protein engineering. Engineering antibodies to enhance properties such as binding affinity and stability plays an essential role in therapeutic applications. Here we report a method, multiplex navigation of antibody structure (MINAS), that combines a CRISPR/Cas9-based trackable editing method and

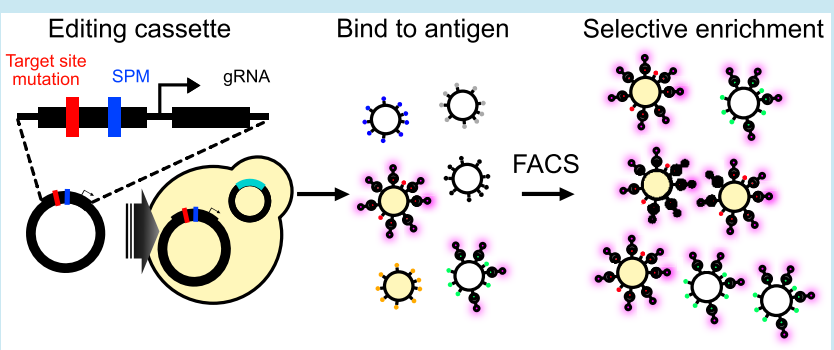
fluorescent-activated cell sorting (FACS) of yeast-displayed libraries. We designed mutations in all of the complementarity-determining and framework regions of a well-characterized scFv antibody and mapped the contribution of these regions to enhanced properties. We identified specific mutants that showed higher binding affinities up to 100-fold compared to the wild-type. This study expands the applicability of CRISPR/Cas9-based trackable protein engineering by combining it with a surface display platform.

KEYWORDS: CRISPR/Cas9, Saccharomyces cerevisiae, yeast display, fluorescent-activated cell sorting, antibody engineering

$\mathrm{T}$ he ability to engineer antibodies with enhanced properties such as binding affinity and stability has had a significant impact on therapeutic applications of antibodies. ${ }^{1}$ Antibody engineering is often performed by targeted mutagenesis or directed evolution using high-throughput screening of phage or yeast display libraries. ${ }^{2}$ Because strategies for generating sequence diversity by random mutagenesis can substitute only limited numbers of amino acids in the targeted position, these approaches might miss other regions that may also affect function that is not easily predicted. ${ }^{3}$ The generation of libraries for searching the mutational landscape has been demonstrated using a PCR-based approach with mutagenic oligos. ${ }^{4,5}$ However, such methods have limitations. Specifically, when a larger backbone plasmid was used to construct libraries, transformation efficiencies were much lower. In addition, increasing the size of the insert to be mutated may cause challenges with sequencing depth and cost. Also, if the backbone plasmid has multiple nicking sites (BbvCI restriction sites) with opposite orientations, both strands of the plasmid will be nicked during the first BbvCI digest, resulting in the degradation of the whole plasmid by exonuclease III.

Many of these limitations are overcome by using CREATE (CRISPR-EnAbled Trackable genome Engineering), an advanced approach based on CRISPR/Cas9 editing and barcode tracking that enables multiplex editing and mapping at the genome scale. ${ }^{6}$ Recent studies employed this approach for high-throughput genome-wide engineering in yeast. $^{7-9}$ These studies have led to new insights into genotypephenotype correlations in yeast as well as bacteria. Here, we report the development of a method, multiplex navigation of antibody structure (MINAS), that combines CRISPR/Cas9based trackable editing and fluorescent-activated cell sorting (FACS) of yeast display libraries. Array-based synthesis of editing cassettes allows for the pooled cloning of plasmid libraries. Each cassette contains (1) a gRNA targeting the antibody, and (2) a dsDNA repair template (homology arm) that introduces designed mutations at the target site (Figure 1a). Following editing with the libraries, the frequency of the designed mutations can be tracked by deep sequencing of the editing plasmids that act as genetic barcodes (Figure $1 \mathrm{~b}$ ). We used this approach for multiplex editing of the complementarity-determining (CDR) and framework (FWR) regions of a single-chain variable fragment ( $\mathrm{scFv}$ ) antibody; however, MINAS can be applied to any region in any antibody to introduce up to hundreds of thousands of mutations and map the effects of those mutations on desired phenotypes.

The ability to edit with high precision is one of the essential factors required in order to apply MINAS to antibody engineering. Thus, we measured editing efficiencies of MINAS cassettes with varying homology arm lengths (48-

Received: March 24, 2020

Published: June 18, 2020 
Pursuant to the DOE Public Access Plan, this document represents the authors' peer-reviewed, accepted manuscript. The published version of the article is available from the relevant publisher.

a

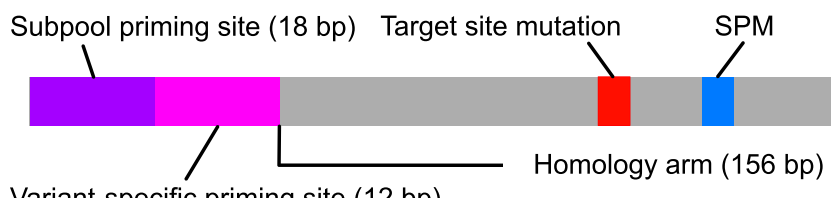

Variant-specific priming site (12 bp)

b
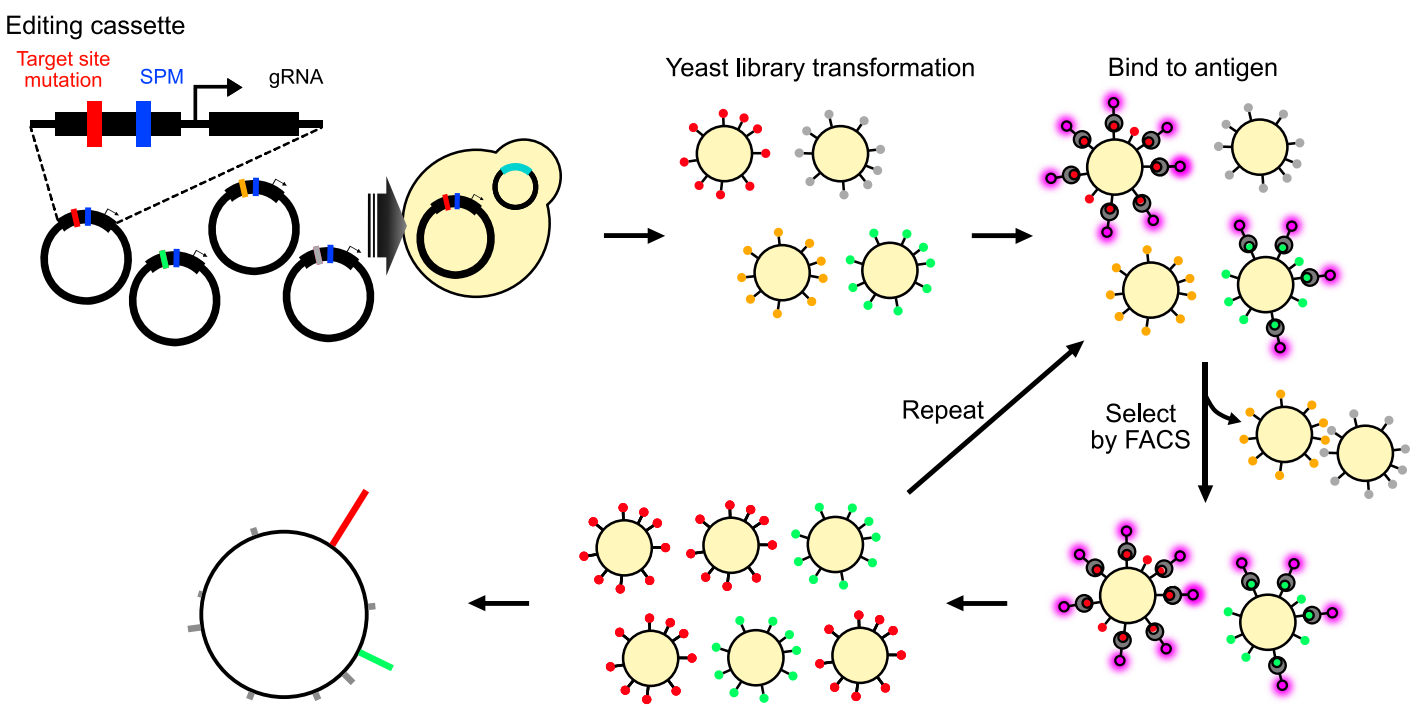<smiles>CC1CC(C)C(C2CC(C)C(C)CC2C)CC1C</smiles>
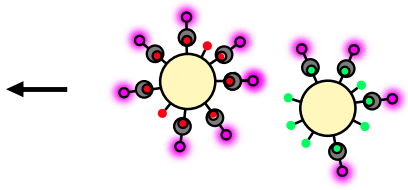

C
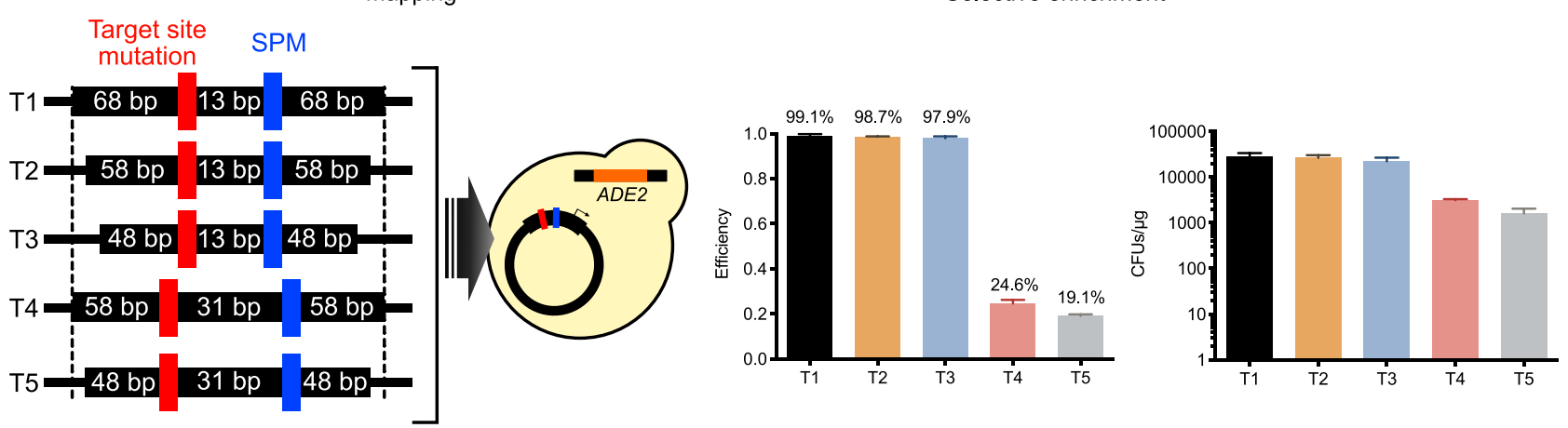

d
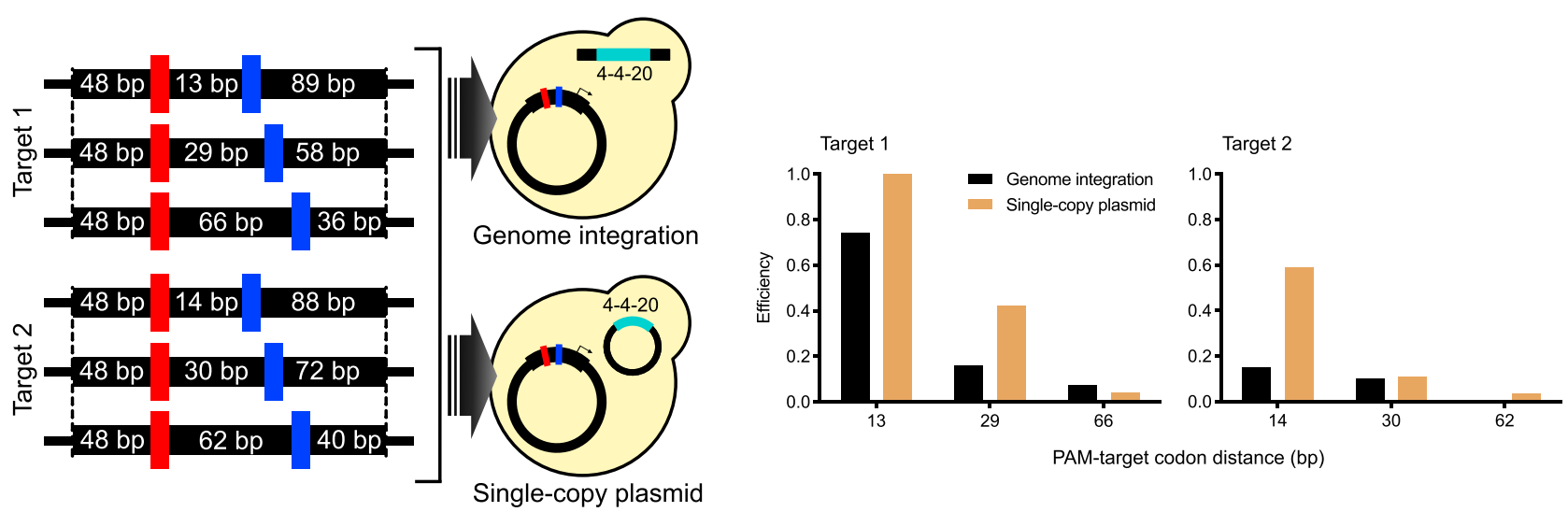

Figure 1. MINAS library construction and selection. (a) Design of the MINAS cassette. (b) The workflow of the strategy. A designed cassette is composed of a homology arm, including the target site mutation, a synonymous PAM mutation (SPM), and the corresponding gRNA. The editing cassettes are cloned in multiplex into a backbone plasmid harboring Cas9. Yeast strains expressing the target antibody are transformed with the library plasmids. Yeast displaying antibodies with various affinities to the antigen are isolated and enriched by iterative rounds of FACS. Following selection, the library plasmids are PCR-amplified, and the frequency of each plasmid before and after selection is determined by deep sequencing. Enrichment scores for each mutant caused by the corresponding cassette are calculated as the $\log _{2}$ ratio of frequencies between postselection and preselection. (c) Editing efficiency and the number of CFUs/ $\mu$ g DNA using editing cassettes with various homology arm lengths and distances between the PAM and target site. (d) Comparison of editing efficiency between genome integration and single-copy plasmid platforms for 4-4-20 expression. 
Pursuant to the DOE Public Access Plan, this document represents the authors' peer-reviewed, accepted manuscript. The published version of the article is available from the relevant publisher.

a

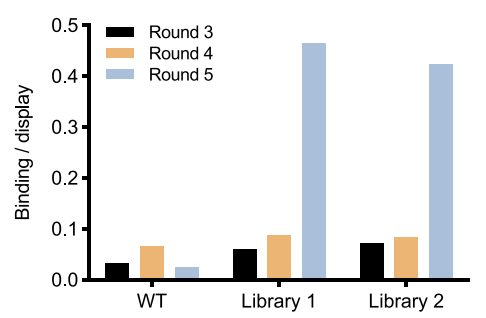

C

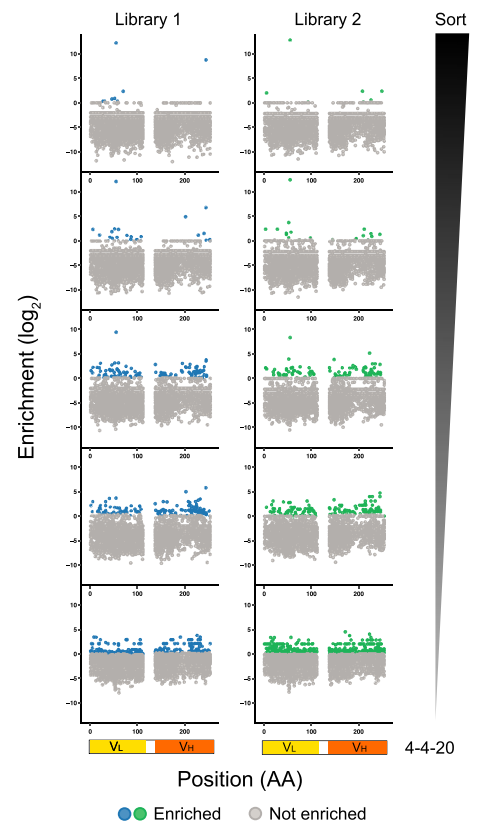

g

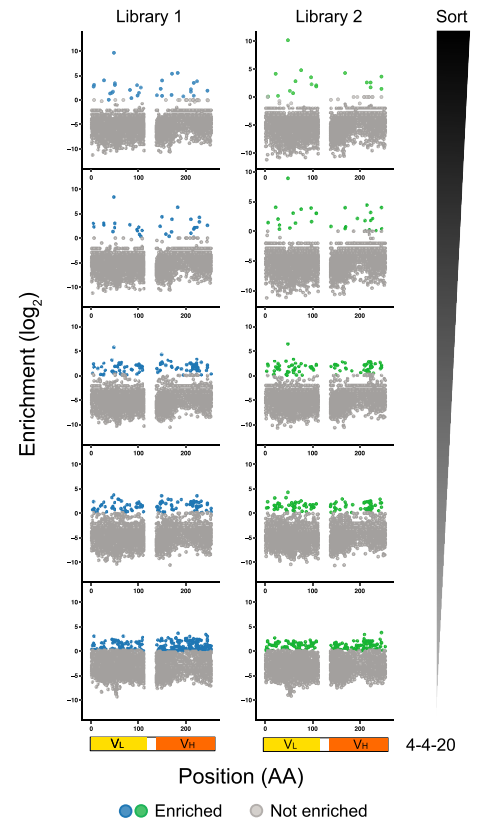

b

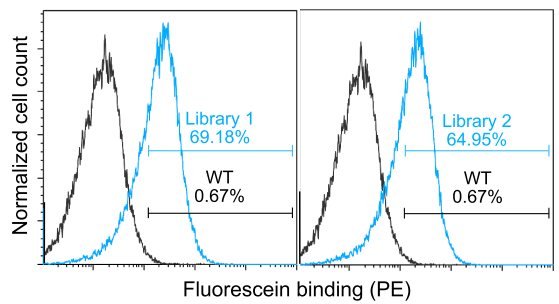

d

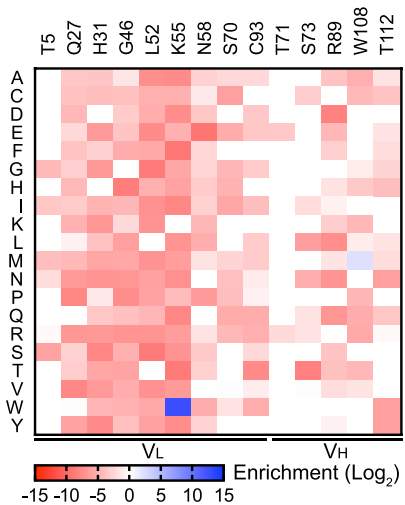

e

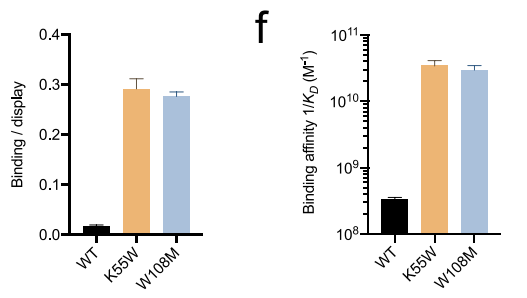

h
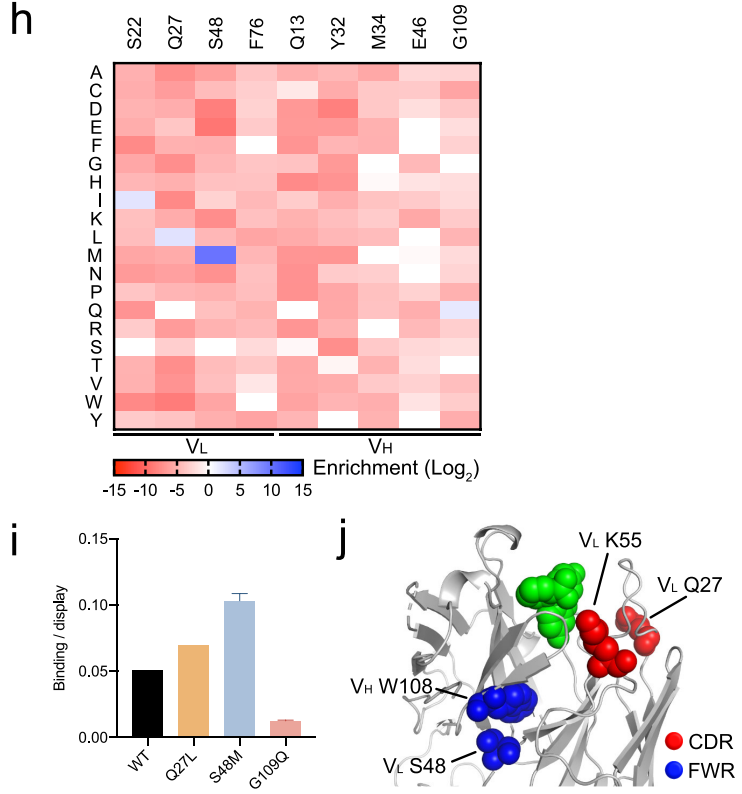

Figure 2. Enrichment analysis of library cells for improved binding affinity $(a-f)$ and low $\mathrm{pH}$ stability ( $\mathrm{g}-\mathrm{i}$ ). (a) The median fluorescence intensity of the binding signal $\left(\mathrm{MFI}_{\text {Binding }}\right)$ was normalized to the median fluorescence intensity of the display signal $\left(\mathrm{MFI}_{\text {Display }}\right)$. The normalized median fluorescence intensity (binding/display $=\mathrm{MFI}_{\text {Binding }} / \mathrm{MFI}_{\text {Display }}$ ) showed progressive enrichment of mutants via FACS for improved binding affinities. (b) Histogram of library fluorescein binding, showing preselection (black) and after five rounds of selection for improved binding affinities (blue). (c,g) Mapping of enrichment across the positions targeted in the 4-4-20 antibody. (d,h) Heatmap showing the enrichment of MINAS cassettes after five rounds of selection. All amino acid residues found in two biological replicates are shown with the average of the enrichment scores. (e) The reconstructed K55W and W108M variants exhibited higher normalized median fluorescence intensity (binding/display $=\mathrm{MFI}_{\text {Binding }} / \mathrm{MFI}_{\text {Display }}$ ) values with binding competition than the wild-type, corresponding to their respective $K_{\mathrm{D}}$ values (f). (i) For the stability of 
Figure 2. continued

the 4-4-20 antibody under acidic conditions, among the top three variants, the S48M and the Q27L mutants showed higher binding/display values compared to the wild-type. The values represent the means of three independent experiments. (j) Structure of the 4-4-20 antibody in complex with fluorescein (green). The highly enriched mutations for both binding affinity and low pH stability are shown in red (CDR) and blue (FWR). PDB code 1FLR.

$68 \mathrm{bp}$ ) and varying distances between the PAM and target site $(13-31 \mathrm{bp})$. For these tests, we inactivated the ADE2 gene by introducing point mutations to convert codons 10 and 11 to TAATAA stop codons. The editing results were monitored by a white to red colony color change and confirmed by Sanger sequencing. The editing efficiency was $99.1 \%$ when the homology arm was longer ( $68 \mathrm{bp}$ ) and the distance between the PAM and target site was shorter $(13 \mathrm{bp})$, consistent with previous studies. 6,9 The effect of the distance between the PAM and target site on editing efficiency was more significant than the length of the homology arm (Figure 1c). Also, we observed a significant increase in transformation efficiency when shorter distances between the PAM and target site were used, suggesting that high-efficiency editing and transformation are possible when cassettes are designed using these criteria (Figure 1c).

We next tested the editing efficiencies in two different antibody expression systems when MINAS cassettes with various distances between the PAM and target site (13-66 bp) were used. We used a well-characterized antibody-antigen system, the 4-4-20 scFv antibody which binds fluorescein. First, the 4-4-20 antibody expression cassette was cloned into the yeast integration vector pRS404, and the resulting plasmid was integrated into the TRP1 locus of Saccharomyces cerevisiae strain EBY100. Second, a single-copy plasmid expressing the 4-4-20 antibody, pCT302, was transformed into the EBY100 strain to express 4-4-20 from a plasmid rather than integrating it into the genome. Next, we synthesized editing cassettes targeting two different codons. After transforming the editing plasmids into yeast harboring each of the 4-4-20 antibody expression platforms, the editing efficiencies were assessed by sequencing both the plasmid barcodes and the targeting sites from 20 to 27 randomly chosen colonies. For the first editing cassette, which targeted codon 67 in the variable light chain $\left(\mathrm{V}_{\mathrm{L}}\right)$ region, the editing efficiencies in the integration platform ranged from 7 to $74 \%$, whereas those in the single-copy plasmid platform ranged from 4 to $100 \%$ depending on the distance between the PAM and target site. Although the editing efficiencies for the second editing cassette, which targeted codon 5 in the variable heavy chain $\left(V_{H}\right)$ region, were lower than those for the first target, the single-copy plasmid platform exhibited higher editing efficiencies than the genome integration platform overall (Figure 1d). Therefore, the single-copy plasmid system was employed for the expression of the 4-4-20 antibody.

To test the feasibility of MINAS as a tool for antibody engineering, we designed 4580 mutations targeting all $\mathrm{V}_{\mathrm{L}}(112$ aa) and $\mathrm{V}_{\mathrm{H}}(117 \mathrm{aa})$ domains in the 4-4-20 antibody (Supplementary Figure 1). A full codon saturation mutagenesis library was introduced by replacing the target residue with the remaining 19 amino acids using the most frequent codons; a stop codon was also included as a negative control. The MINAS libraries were constructed in Escherichia coli as previously described, ${ }^{9}$ and the accuracy of plasmid libraries was assessed by sequencing the PCR-amplified editing cassettes from randomly picked colonies. We observed that
$55 \%$ (23/42) matched the cassette design with $100 \%$ identity, $12 \%$ contained a $1-2 \mathrm{bp}$ insertion/deletion (indel) (5/42), and $33 \%(14 / 42)$ were chimeric cassettes. All the perfectly matched cassettes were unique, indicating that the libraries were effectively prepared with mutational diversity. Discrepancies in the plasmid libraries may have arisen during synthesis, PCR-amplification, or Gibson assembly. The library plasmids were extracted and retransformed into $S$. cerevisiae EBY100 harboring pCT302.

We first demonstrated the MINAS approach by mapping mutations that confer increased binding affinity. The resulting yeast libraries were incubated with biotinylated fluorescein and then exposed to a competitor, 5-aminofluorescein, causing a loss in antigen-binding activity. Cells displaying 4-4-20 mutants that bound fluorescein more strongly than wild-type cells after the binding competition were isolated by FACS (Figure 1b). Antibody-bound fluorescein was visualized using PE dye, and the amount of surface-displayed antibody was separately visualized using Alexa Fluor 488 (c-Myc). We reasoned that designer mutations conferring improved affinity would outcompete the 5-aminofluorescein and thereby be enriched through repeated FACS sorting of the top $0.1-5 \%$ of antigen-binding c-Myc positive clones. Sequencing the barcode of the editing cassettes before and after sorting in the presence of the competitor enabled parallel tracking of mutants with enrichment and mapping of their contribution to desired phenotypes. To ensure high editing efficiency and adequate mutant identification, only reads that matched a designed cassette with an identity of greater than $99 \%$ were used. Deep sequencing of the initial yeast population that was transformed with MINAS cassettes prior to sorting showed $78-81 \%$ of the designs were contained in the library pools across two biological replicates. Sorting the pools with increasing stringency of sort gates resulted in sharply increased enriched populations of library cells (Figure 2a). After five rounds of selection (Figure 2b), plasmids in the enriched populations were isolated, and the regions containing the barcode of the editing cassettes were sequenced.

When mapped at single amino acid resolution, we observed significantly enriched mutations in both CDR and FWR regions across all targeted areas based on the average enrichment scores obtained in the two biological replicates (Figure 2c,d). Of the two top mutations we identified, the $\mathrm{K} 55 \mathrm{~W}$ mutation in CDR2 of the $\mathrm{V}_{\mathrm{L}}$ domain was previously unreported, and the $\mathrm{W} 108 \mathrm{M}$ mutation in the framework region of the $\mathrm{V}_{\mathrm{H}}$ domain was described previously ${ }^{10}$ (Figure $2 \mathrm{j}$ ). We individually reconstructed these two 4-4-20 variants and tested their binding affinities using the same competition process as described above. Both reconstructed variants exhibited more than 16-fold higher binding/display values, consistent with their respective enrichment profiles (Figure 2e). Next, we determined $K_{D}$ values for individual variants by measuring binding curves as described by Chao et al. ${ }^{11}$ In addition to wild-type 4-4-20, K55W, and W108M, we assayed the 4 M5.3 variant from Boder et al. ${ }^{10}$ As expected, the 4 M5.3 variant, with 14 accumulated mutations, showed the 
highest affinity $\left(K_{\mathrm{D}}<0.014 \mathrm{nM}\right)$ among the variants, and the $\operatorname{K55W}\left(K_{\mathrm{D}}=0.030 \mathrm{nM}\right)$ and W108M $\left(K_{\mathrm{D}}=0.035 \mathrm{nM}\right)$ mutants achieved roughly a 90 - to 100 -fold improvement in affinity as compared to the wild-type $\left(K_{\mathrm{D}}=3.055 \mathrm{nM}\right)$ (Supplementary Figure 2, Supplementary Table 1, Figure 2f). Amino acid substitutions in proteins can change multiple biochemical properties of the proteins. ${ }^{12}$ For example, mutation(s) may lead to stabilization of intraloop interactions or free energy differences, resulting in binding affinity improvements. ${ }^{13-15}$ Here we show that both a residue in the CDR2 region, which constitutes a paratope, and a residue in the framework region adjacent to CDR3 were influential for affinity. These results suggest that although CDR3 typically contributes the most to antigen specificity, ${ }^{16}$ broader mutational strategies including both CDR and FWR regions are critical in antibody engineering. Furthermore, these results demonstrate the potential ability of the MINAS approach to perform rapid mapping of target antibodies for the identification of novel mutations.

Exposure to low $\mathrm{pH}$ conditions is unavoidable for antibody purification and leads to beneficial effects on viral clearance in the production process. However, when exposed to acidic conditions, antibodies can undergo conformational changes. ${ }^{17}$ Thus, enhanced low $\mathrm{pH}$ stability is an important trait for industrial antibody production. Therefore, we next used MINAS to improve the stability of 4-4-20 under acidic conditions. Yeast expressing 4-4-20 antibody libraries were incubated in buffers with a $\mathrm{pH}$ range of $2-3$ for $1 \mathrm{~h}$ at room temperature before antigen binding, and labeling reactions and FACS proceeded in the same manner as for binding competition. We found that there was no significant difference in low $\mathrm{pH}$ stability between preselection and after five rounds of selection across two biological replicates. Although we did not see an obvious difference in pre- and postselection libraries, we nonetheless did enrichment analysis in an attempt to map mutations that might confer low $\mathrm{pH}$ stability (Figure $2 \mathrm{~g}, \mathrm{~h})$. To confirm the enrichment analysis, we reconstructed the top three variants (Q27L, S48M, and G109Q) from the fifth-round of selection and examined their binding affinities under acidic conditions by measuring binding/display values. Although we did not directly measure stability with the reconstructed variants, it has been shown that measuring binding affinity by yeast display correlates very well to other measurements of stability done with soluble scFvs. ${ }^{18}$ Among the three variants, the S48M and the Q27L mutants, which were the first and second most enriched in both replicates, showed 2- and 1.4-fold higher binding affinity compared to the wild-type, respectively, consistent with their enrichment scores (Figure 2i,j). Although we could not observe a significant increase in enriched populations from the library cells during FACS, the dominant selection winners exhibited the desired phenotypes. These identified mutations might be involved in thermodynamic stability, perhaps through resistance to acid aggregation. ${ }^{19}$

Recent advances in next-generation sequencing and CRISPR/Cas9-based editing have allowed more thorough investigations of target proteins at single-nucleotide resolution. $^{20-22}$ Yeast surface display enables quantitative library analysis and screening by flow cytometry and is a powerful platform for engineering antibodies. ${ }^{23}$ In this study, we demonstrate a novel CRISPR/Cas9-based approach for rapid and trackable antibody engineering based on yeast surface display. By combining CRISPR/Cas9 gene editing with multiplexed oligo synthesis and deep sequencing, we were able to evaluate the contribution of each mutation to the desired phenotypes of the antibody with trackable highthroughput mapping by a simple workflow.

Although we were able to track the enrichment of library variants through multiplex fitness mapping for antibody engineering, some limitations were identified in our approach. It is necessary to overcome the low editing efficiency on a library scale and unintended mutations arising during library preparation by improving the CRISPR/Cas9 machinery ${ }^{24,25}$ and design/assembly process. ${ }^{12,26}$ Sorting the pools with increasing stringency of sort gates might result in the convergence of mutations. However, using a single sort makes it difficult to identify real positive variants because there are too many variants to test (Supplementary Table 2). To further enhance the MINAS approach, magnetic-activated cell sorting (MACS) can be used as the first step in the workflow to reduce false-positive mutants. Also, modulating parameters for selection pressures, including sort gates and competition times, could enable quantification with enhanced diversity. Despite these limitations, CREATE libraries can be built with $>100000$ single nucleotide polymorphisms (SNPs) which can be mapped to identify mutations that confer phenotypes of interest in parallel. ${ }^{6}$ Using these larger libraries, MINAS would allow a full codon saturation mutagenesis of an entire antibody regardless of the size of the antibody. Extension of this technology might be adopted for highthroughput mapping of other antibody-antigen interactions of interest such as epitope mapping.

\section{ASSOCIATED CONTENT}

\section{(3) Supporting Information}

The Supporting Information is available free of charge at https://pubs.acs.org/doi/10.1021/acssynbio.0c00159.

Supplementary methods, yeast display construct, binding titration curves of yeast displayed 4-4-20 and mutant scFvs, $K_{\mathrm{D}}$ measured using flow cytometry, the number of mutations with $\log _{2}$ enrichment greater than 0 after selection, strains, and plasmids used in this study, library plasmid construction primers, gBlock sequences used in this study (PDF)

\section{AUTHOR INFORMATION}

\section{Corresponding Author}

Ryan T. Gill - Renewable and Sustainable Energy Institute (RASEI), University of Colorado Boulder, Boulder, Colorado 80303, United States; Novo Nordisk Foundation Center for Biosustainability, Danish Technical University, Lyngby, Denmark; ○orcid.org/0000-0003-2062-303X; Email: rtg@ biosustain.dtu.dk

\section{Authors}

Eun Joong Oh - Renewable and Sustainable Energy Institute (RASEI), University of Colorado Boulder, Boulder, Colorado 80303, United States; -1 orcid.org/0000-0001-7145-0752

Rongming Liu - Renewable and Sustainable Energy Institute (RASEI), University of Colorado Boulder, Boulder, Colorado 80303, United States; 1 orcid.org/0000-0001-5014-8976

Liya Liang - Renewable and Sustainable Energy Institute (RASEI), University of Colorado Boulder, Boulder, Colorado 80303, United States 
Emily F. Freed - Renewable and Sustainable Energy Institute (RASEI), University of Colorado Boulder, Boulder, Colorado 80303, United States

Carrie A. Eckert - Renewable and Sustainable Energy Institute (RASEI), University of Colorado Boulder, Boulder, Colorado 80303, United States; National Renewable Energy Laboratory (NREL), Bioscience Center, Golden, Colorado 80401, United States; orcid.org/0000-0003-4201-2926

Complete contact information is available at: https://pubs.acs.org/10.1021/acssynbio.0c00159

\section{Author Contributions}

E.J.O. and R.T.G. designed research. E.J.O. performed research and analyzed data. Sequencing data analysis was done by R.L. and E.F.F. L.L. helped with cloning. C.A.E. aided the design of experiments. E.J.O. and R.T.G. wrote the manuscript. E.F.F. and C.A.E. reviewed and edited the manuscript. All authors read and approved the paper.

\section{Notes}

The authors declare the following competing financial interest(s): The author R.T.G. has financial interest in the company Inscripta, Inc., which is commercializing the technology used in this manuscript. The remaining authors declare no competing interests.

\section{ACKNOWLEDGMENTS}

We thank Karen Helm (Cancer Center Support Grant (P30CA046934) and the Skin Diseases Research Cores Grant (P30AR057212)) for assistance and support with FACS runs performed in this study. This work was supported by the US Department of Energy (Grant DE-SC0018368) and Inscripta, Inc.

\section{REFERENCES}

(1) Chiu, M. L., and Gilliland, G. L. (2016) Engineering antibody therapeutics. Curr. Opin. Struct. Biol. 38, 163-173.

(2) Hoogenboom, H. R. (2005) Selecting and screening recombinant antibody libraries. Nat. Biotechnol. 23, 1105-1116.

(3) Kazlauskas, R. J., and Bornscheuer, U. T. (2009) Finding better protein engineering strategies. Nat. Chem. Biol. 5, 526-529.

(4) Firnberg, E., and Ostermeier, M. (2012) PFunkel: efficient, expansive, user-defined mutagenesis. PLoS One 7, No. e52031.

(5) Wrenbeck, E. E., Klesmith, J. R., Stapleton, J. A., Adeniran, A., Tyo, K. E. J., and Whitehead, T. A. (2016) Plasmid-based one-pot saturation mutagenesis. Nat. Methods 13, 928-930.

(6) Garst, A. D., Bassalo, M. C., Pines, G., Lynch, S. A., HalwegEdwards, A. L., Liu, R., Liang, L., Wang, Z., Zeitoun, R., Alexander, W. G., and Gill, R. T. (2017) Genome-wide mapping of mutations at single-nucleotide resolution for protein, metabolic and genome engineering. Nat. Biotechnol. 35, 48-55.

(7) Bao, Z., HamediRad, M., Xue, P., Xiao, H., Tasan, I., Chao, R., Liang, J., and Zhao, H. (2018) Genome-scale engineering of Saccharomyces cerevisiae with single-nucleotide precision. Nat. Biotechnol. 36, 505-508.

(8) Guo, X., Chavez, A., Tung, A., Chan, Y., Kaas, C., Yin, Y., Cecchi, R., Garnier, S. L., Kelsic, E. D., Schubert, M., DiCarlo, J. E., Collins, J. J., and Church, G. M. (2018) High-throughput creation and functional profiling of DNA sequence variant libraries using CRISPR-Cas9 in yeast. Nat. Biotechnol. 36, 540-546.

(9) Liu, R., Liang, L., Choudhury, A., Garst, A. D., Eckert, C. A., Oh, E. J., Winkler, J., and Gill, R. T. (2019) Multiplex navigation of global regulatory networks (MINR) in yeast for improved ethanol tolerance and production. Metab. Eng. 51, 50-58.

(10) Boder, E. T., Midelfort, K. S., and Wittrup, K. D. (2000) Directed evolution of antibody fragments with monovalent femtomolar antigen-binding affinity. Proc. Natl. Acad. Sci. U. S. A. 97, 10701-10705.

(11) Chao, G., Lau, W. L., Hackel, B. J., Sazinsky, S. L., Lippow, S. M., and Wittrup, K. D. (2006) Isolating and engineering human antibodies using yeast surface display. Nat. Protoc. 1, 755-768.

(12) Adams, R. M., Mora, T., Walczak, A. M., and Kinney, J. B. (2016) Measuring the sequence-affinity landscape of antibodies with massively parallel titration curves. eLife 5, 23156 DOI: 10.7554/ eLife.23156.

(13) Midelfort, K. S., Hernandez, H. H., Lippow, S. M., Tidor, B., Drennan, C. L., and Wittrup, K. D. (2004) Substantial energetic improvement with minimal structural perturbation in a high affinity mutant antibody. J. Mol. Biol. 343, 685-701.

(14) Midelfort, K. S., and Wittrup, K. D. (2006) Context-dependent mutations predominate in an engineered high-affinity single chain antibody fragment. Protein Sci. 15, 324-334.

(15) Chlewicki, L. K., Holler, P. D., Monti, B. C., Clutter, M. R., and Kranz, D. M. (2005) High-affinity, peptide-specific T cell receptors can be generated by mutations in CDR1, CDR2 or CDR3. J. Mol. Biol. 346, 223-239.

(16) Sundberg, E. J., and Mariuzza, R. A. (2002) Molecular recognition in antibody-antigen complexes. Adv. Protein Chem. 61, 119-160.

(17) Ejima, D., Tsumoto, K., Fukada, H., Yumioka, R., Nagase, K., Arakawa, T., and Philo, J. S. (2007) Effects of acid exposure on the conformation, stability, and aggregation of monoclonal antibodies. Proteins: Struct., Funct., Genet. 66, 954-962.

(18) Orr, B. A., Carr, L. M., Wittrup, K. D., Roy, E. J., and Kranz, D. M. (2003) Rapid method for measuring scFv thermal stability by yeast surface display. Biotechnol. Prog. 19, 631-638.

(19) Famm, K., Hansen, L., Christ, D., and Winter, G. (2008) Thermodynamically stable aggregation-resistant antibody domains through directed evolution. J. Mol. Biol. 376, 926-931.

(20) Mason, D. M., Weber, C. R., Parola, C., Meng, S. M., Greiff, V., Kelton, W. J., and Reddy, S. T. (2018) High-throughput antibody engineering in mammalian cells by CRISPR/Cas9-mediated homology-directed mutagenesis. Nucleic Acids Res. 46, 7436-7449.

(21) Devilder, M.-C., Moyon, M., Gautreau-Rolland, L., Navet, B., Perroteau, J., Delbos, F., Gesnel, M.-C., Breathnach, R., and Saulquin, X. (2019) Ex vivo evolution of human antibodies by CRISPR-X: from a naive $\mathrm{B}$ cell repertoire to affinity matured antibodies. $B M C$ Biotechnol. 19, 14.

(22) Simon, A. J., d'Oelsnitz, S., and Ellington, A. D. (2019) Synthetic evolution. Nat. Biotechnol. 37, 730-743.

(23) Gai, S. A., and Wittrup, K. D. (2007) Yeast surface display for protein engineering and characterization. Curr. Opin. Struct. Biol. 17, $467-473$.

(24) Slaymaker, I. M., Gao, L., Zetsche, B., Scott, D. A., Yan, W. X., and Zhang, F. (2016) Rationally engineered Cas9 nucleases with improved specificity. Science 351, 84-88.

(25) Hu, J. H., Miller, S. M., Geurts, M. H., Tang, W., Chen, L., Sun, N., Zeina, C. M., Gao, X., Rees, H. A., Lin, Z., and Liu, D. R. (2018) Evolved Cas9 variants with broad PAM compatibility and high DNA specificity. Nature 556, 57-63.

(26) Kinney, J. B., Murugan, A., Callan, C. G., and Cox, E. C. (2010) Using deep sequencing to characterize the biophysical mechanism of a transcriptional regulatory sequence. Proc. Natl. Acad. Sci. U. S. A. 107, 9158-9163. 